UVX $2010(2011) 35-40$

DOI: $10.1051 / \mathrm{uvx} / 2011005$

(c) Owned by the authors, published by EDP Sciences, 2011

\title{
Optiques pour les impulsions attosecondes
}

\author{
C. Bourassin-Bouchet ${ }^{1, *}$, Z. Diveki ${ }^{2}$, S. de Rossi ${ }^{1}$, E. English ${ }^{2}$, \\ E. Meltchakov ${ }^{1}$, O. Gobert ${ }^{2}$, D. Guénot ${ }^{2}$, B. Carré $^{2}$, F. Delmotte ${ }^{1}$, \\ T. Ruchon ${ }^{2}$, P. Chavel ${ }^{1}$ et P. Salières ${ }^{2}$ \\ ${ }^{1}$ Laboratoire Charles Fabry, Institut d'Optique, Université Paris-Sud, CNRS, 2 avenue \\ Augustin Fresnel, 91127 Palaiseau, France \\ ${ }^{2}$ CEA-Saclay, IRAMIS, Service des Photons, Atomes et Molécules, 91191 Gif-sur-Yvette, \\ France
}

\begin{abstract}
Résumé. Nous présentons les différents aspects de la conception de composants optiques de type miroirs multicouches, pour les sources d'impulsions extrême ultraviolet (EUV) attosecondes. Pour manipuler efficacement ces impulsions, ces optiques doivent pouvoir transporter un spectre très large tout en conservant la relation de phase existante entre les composantes spectrales. De plus, ces impulsions ultrabrèves s'avèrent extrêmement sensibles aux aberrations optiques, ces dernières ayant pour effet de déformer et étirer les impulsions attosecondes.
\end{abstract}

\section{INTRODUCTION}

En 2001 furent générées les premières impulsions lumineuses attosecondes, sous la forme d'un train d'impulsions de 250 as [1], mais également sous la forme d'impulsions isolées de 650 as [2]. Grâce à l'optimisation des différentes sources d'impulsions attosecondes dans le monde, les durées ont pu être divisées par un facteur proche de dix en une décennie, le record de durée étant à l'heure actuelle de 63 as [3] pour des impulsions émises sous forme de train, et de 80 as pour des impulsions isolées [4].

Le processus physique à l'origine de ces impulsions est appelé génération d'harmoniques d'ordre élevé. En focalisant une impulsion infrarouge femtoseconde suffisamment intense dans un gaz rare, on obtient des harmoniques de la fréquence infrarouge fondamentale [5,6] dans le domaine EUV, typiquement entre la dizaine et la centaine d'électron-volts. Une propriété remarquable de ces harmoniques est qu'il existe une relation de phase spectrale entre elles. En d'autres termes, lorsqu'elles sont générées, ces harmoniques sont suffisamment synchronisées pour pouvoir se superposer dans le temps et donner des impulsions attosecondes. Cependant, cette synchronisation temporelle n'est pas parfaite. En effet, il a été montré [7] que durant le processus de génération, les premiers ordres harmoniques sont générés légèrement plus tôt que les harmoniques d'ordres plus élevés, cette désynchronisation étirant les impulsions attosecondes. Ce phénomène est appelé l'attochirp.

L'une des méthodes les plus simples et les plus répandues pour caractériser temporellement ces impulsions est la méthode RABITT (Reconstruction of Attosecond Beating by Interference of Twophoton Transitions) [8]. Le train d'impulsions attosecondes ainsi qu'une impulsion femtoseconde infrarouge faiblement intense sont focalisés dans un jet de gaz rare. Sous l'effet des photons EUV, les atomes de gaz sont ionisés, créant des photoélectrons. En mesurant l'énergie de ces photoélectrons avec un spectromètre à temps de vol, en fonction du retard imposé entre l'impulsion infrarouge et le train d'impulsions attosecondes, on obtient une trace RABITT. Cette trace contient à la fois le spectre des impulsions attosecondes, et leur phase spectrale, c'est à dire la quantité donnant la synchronisation

*e-mail : charles.bourassin-bouchet@institutoptique.fr

This is an Open Access article distributed under the terms of the Creative Commons Attribution-Noncommercial License 3.0, which permits unrestricted use, distribution, and reproduction in any noncommercial medium, provided the original work is properly cited. 
relative des harmoniques. Or la connaissance du spectre et de la phase spectrale est totalement suffisante pour caractériser des impulsions ultrabrèves, puisqu'une simple transformée de Fourier suffit alors pour obtenir la structure temporelle de l'impulsion.

Avec le développement continu des "lignes de lumière" attosecondes, l'étude et le développement de composants optiques aptes à manipuler ces impulsions deviennent des points cruciaux. De telles optiques doivent contrôler le spectre, mais également la phase spectrale, ainsi que la phase spatiale de ces impulsions. Cela peut être obtenu grâce à des miroirs multicouches, qui sont des composants déjà répandus dans le domaine EUV.

\section{TRANSPORT ET COMPRESSION D'IMPULSIONS : OPTIQUES MULTICOUCHES}

\subsection{Position du problème}

Un miroir multicouches consiste en un simple empilement de couches minces faites de différents matériaux (voir Fig. 1(a))). Le molybdène (Mo), le silicium (Si), voire le carbure de Bore (B4C) sont généralement utilisés dans le domaine EUV. Le choix de la structure de l'empilement (matériaux, épaisseurs et ordre des couches) fixe le comportement spectral du miroir.

Dans le cas des impulsions attosecondes, la première condition à remplir pour réfléchir une impulsion sans la détruire est de conserver, lors de la réflexion, le spectre complet de l'impulsion, large de plusieurs dizaines d'électron-volts. Le miroir doit donc avoir une très large bande passante. Cela est possible par l'utilisation de structures multicouches totalement apériodiques [9], obtenues à l'aide d'algorithmes d'optimisation complexes. Le second point est le contrôle de la phase spectrale réfléchie par le miroir, c'est à dire la capacité du miroir à plus ou moins désynchroniser les harmoniques les unes par rapport aux autres. En particulier, on pourrait imaginer compenser la désynchronisation des harmoniques due à l'attochirp, ce qui permettrait de raccourcir la durée des impulsions lors de la réflexion. Concevoir de tels miroirs revient à ajouter des contraintes supplémentaires sur la phase de la structure multicouche lors du processus d'optimisation. Deux possibilités s'offrent alors:

i) Si l'on souhaite réfléchir l'impulsion sans la déformer, le miroir devra avoir une phase spectrale linéaire [10].

ii) Si par contre, on souhaite compenser l'attochirp et resynchroniser les harmoniques grâce au miroir, ce dernier devra être inversement chirpé par rapport à l'impulsion. Sa phase spectrale devra être parabolique convexe [11,12].

\subsection{Design et fabrication des miroirs}

Nous avons optimisé une série de miroirs sur la plage spectrale $35-55 \mathrm{eV}$, où les harmoniques sont généralement intenses, et pour un angle d'incidence de $45^{\circ}$. La réflectivité devait être de plus de $10 \%$, et la phase des miroirs devait être contrôlée sur toute la bande passante. Les résultats des optimisations apparaissent en figure 1:

i) Le miroir A dont les performances théoriques sont représentées en pointillés sur la figure 1(b)) est un miroir à phase spectrale linéaire sur la plage optimisée : c'est un miroir de transport d'impulsions. De plus, sa réflectivité théorique est de plus de $20 \%$ sur quasiment toute la plage $35-55 \mathrm{eV}$.

ii) Le miroir B quant à lui (voir Fig. 1(c))) a été optimisé pour avoir une phase spectrale parabolique convexe : c'est un miroir de compression. Etant donnée la contrainte supplémentaire sur la phase, la réflectivité est moins importante que pour le miroir $\mathrm{A}$, mais reste supérieure à $10 \%$ sur la gamme $35-55 \mathrm{eV}$.

\subsection{Caractérisation des miroirs}

Une fois les structures multicouches connues, on peut fabriquer les miroirs et les caractériser en vérifiant leur réflectivité et leur phase réfléchie. Or caractériser la réflectivité d'un miroir se fait aisément sur 
a)

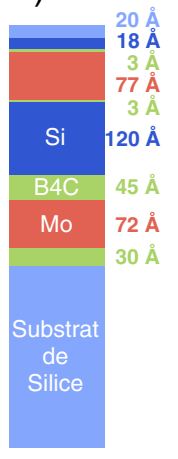

b)

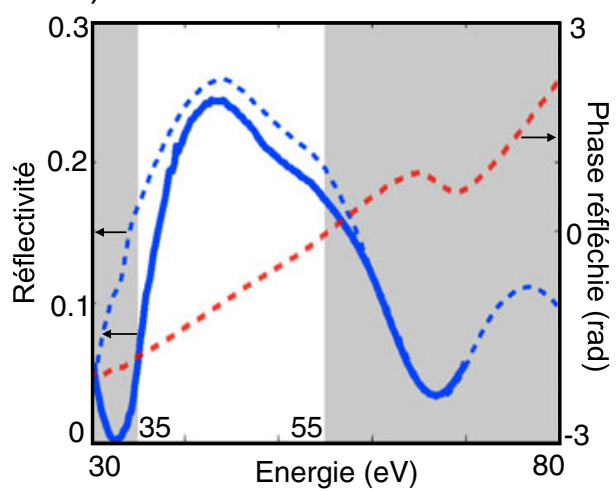

c)

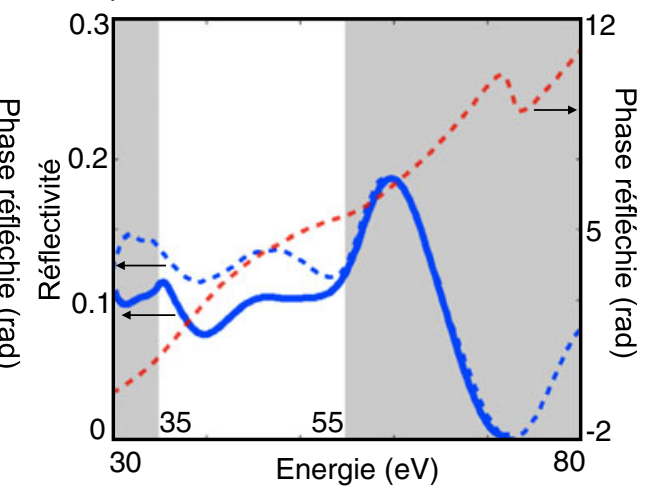

Figure 1. a) Structure multicouche du miroir A. b) et c) Réflectivités théoriques (traits pointillés bleu) et mesurées sur synchrotron (traits continus bleu) et phases réfléchies théoriques (traits pointillés rouge) pour les miroirs A (b) et B (c). La zone non grisée correspond à la bande spectrale 35-55 eV sur laquelle les performances des miroirs ont été optimisées.

la ligne de métrologie d'un synchrotron. Nous avons mesuré la réflectivité des miroirs A et B sur la ligne BEAR du Synchrotron italien ELETTRA. Les résultats sont représentés en traits continus sur les figures 1(b)) et c), l'accord avec les réflectivités théoriques est bon. Les réflectivités mesurées sont légèrement inférieures à celles attendues mais restent toujours au-dessus de la dizaine de pourcent.

La partie la plus délicate reste la mesure de la phase spectrale des miroirs. Pour effectuer une telle caractérisation, le moyen le plus simple est de mesurer avec la méthode RABITT les impulsions attosecondes obtenues avec ou sans réflexion sur un miroir multicouches. En comparant les résultats obtenus dans les deux cas, on peut remonter à la phase spectrale de chaque miroir. Cette caractérisation a été effectuée au CEA Saclay, et a montré un très bon accord entre les phases spectrales théoriques et mesurées [13].

Il est donc possible d'optimiser, de fabriquer et de caractériser totalement ces miroirs multicouches, qui constituent donc une solution pertinente pour manipuler les impulsions attosecondes.

\section{FOCALISATION D'IMPULSIONS : IMPACT DES ABERRATIONS}

\subsection{Position du problème}

Comme expliqué précédemment, la refocalisation des impulsions attosecondes dans un spectromètre à temps de vol est obligatoire pour pouvoir les caractériser. Mais jusqu'à présent, cette refocalisation n'a été effectuée que par des miroirs toriques [3,7], ou des miroirs sphériques hors-axe [1,2,4]. Pour ces optiques, il n'existe pas de conjugaison optique parfaitement stigmatique, si bien que les impulsions seront soumises à des variations de chemins optiques durant la refocalisation. Or une impulsion de 100 as peut être vue comme un mince front de photons EUV épais de $30 \mathrm{~nm}$. Ce constat simple impose une conséquence immédiate : si durant la refocalisation d'une impulsion attoseconde, les photons subissent des variations de chemins optiques de quelques dizaines de nanomètres, alors l'impulsion sera étirée. En d'autres termes, les aberrations des optiques peuvent déformer et rallonger les impulsions attosecondes.

Ce type d'effets est déjà bien connu dans le domaine des impulsions visibles femtosecondes [14-16]. Mais les impulsions attosecondes étant plus brèves, l'impact de aberrations devient d'autant plus critique. 


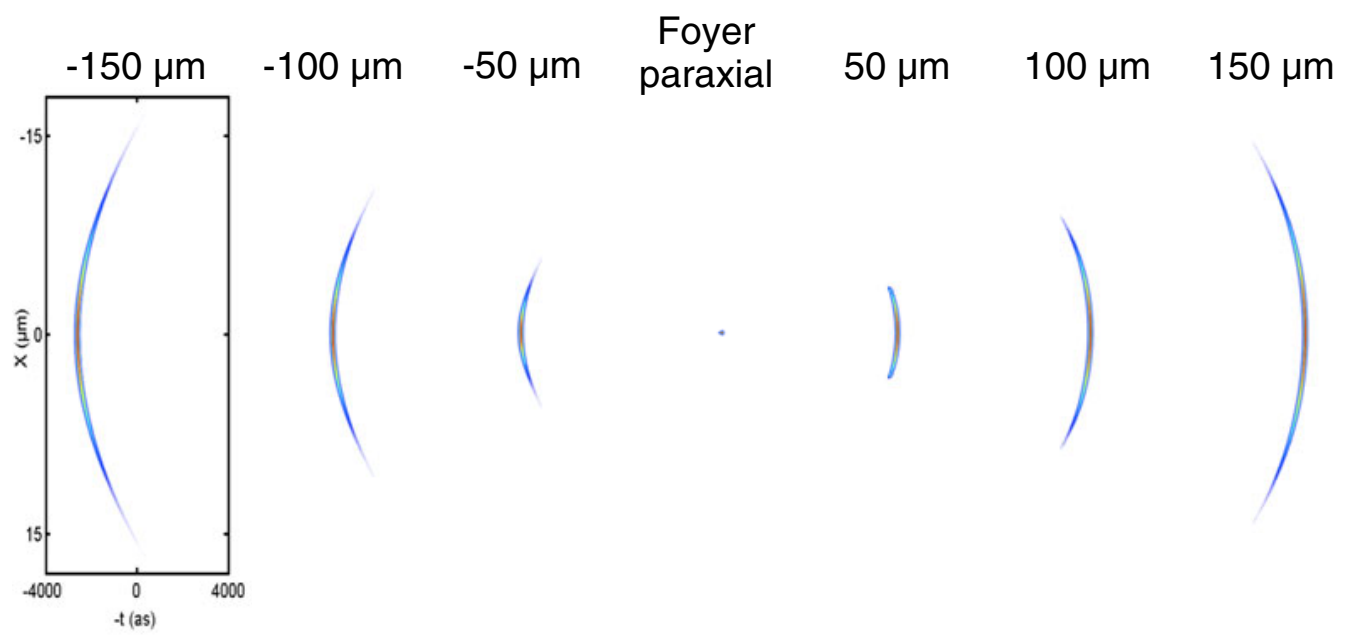

Figure 2. Evolution de l'intensité d'une impulsion attoseconde réfléchie par un miroir parabolique utilisé sur l'axe. Pour différentes position autour du foyer paraxial, l'intensité de l'impulsion (module carré du champ électrique) est représentée en fonction de la position radiale et du temps.

\subsection{Résultats théoriques}

Pour étudier l'influence des aberrations des optiques sur ces impulsions, nous avons développé un code de calcul simulant spatiotemporellement la réflexion d'une impulsion attoseconde sur des optiques couramment utilisées.

Nous avons étudié, dans un premier temps, un miroir parabolique d'une focale de $100 \mathrm{~mm}$, utilisé sur l'axe pour refocaliser des impulsions émises par une source située à $3 \mathrm{~m}$ du miroir. Les conditions initiales choisies pour la source sont détaillées dans [17]. La taille de la tache faite par le faisceau EUV sur le miroir fixe l'ouverture numérique du faisceau refocalisé à 0.04 . Le problème étant à symétrie de révolution, simuler l'impulsion dans un plan radial à l'axe de propagation est suffisant. Les résultats obtenus sont représentés sur la figure 2: les fronts d'impulsions refocalisés sont parfaitement sphériques, indiquant l'absence d'aberrations.

Remplaçons cette fois le miroir parabolique par un miroir sphérique de $100 \mathrm{~mm}$ de focale utilisé avec un angle de champ de $1^{\circ}$ (voir Fig. 3(a))), la source attoseconde utilisée étant la même que pour le miroir parabolique. L'absence de symétrie de révolution oblige à effectuer des simulations dans un volume $(\mathrm{x}, \mathrm{y}, \mathrm{t})$ et non plus seulement dans un plan radial. Les résultats sont représentés sur les figures 3(b)) et c). L'utilisation d'un miroir sphérique avec un faible angle de champ provoque l'apparition de coma et d'aberration sphérique. Du fait de cette dernière, l'évolution de l'impulsion de part et d'autre du foyer paraxial n'est plus symétrique. La coma, quant à elle, provoque l'apparition d'un double front donnant une forme en $\mathrm{V}$ inversé à l'impulsion. Enfin, travailler sur l'axe avec ce miroir sphérique ne réglerait pas tous les problèmes pour autant, puisque seule la coma disparaitrait. Le résultat de la figure 3(b)) deviendrait alors à symétrie de révolution, mais serait toujours marqué par l'aberration sphérique. Ces simulations illustrent donc le fort impact des aberrations sur les impulsions attosecondes.

\section{CONCLUSION}

La conception de composants optiques avancés pour les lignes attosecondes est un point crucial pour permettre à ces sources de se généraliser. En particulier, nous avons optimisé des miroirs multicouches capables de réfléchir sans les détruire des impulsions attosecondes, et leur caractérisation expérimentale complète a été effectuée. Les miroirs multicouches sont donc particulièrement adaptés pour transporter 
a)

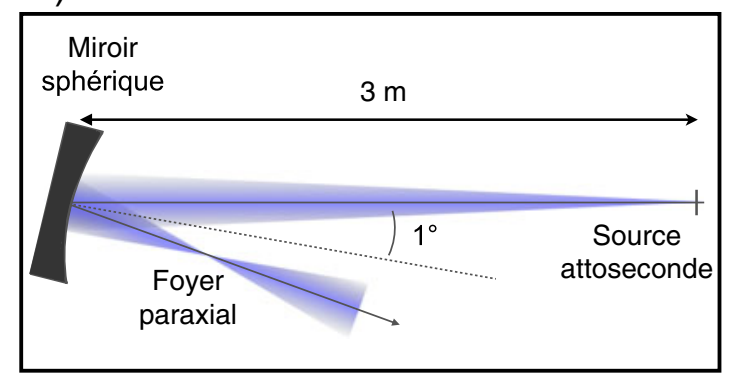

b)
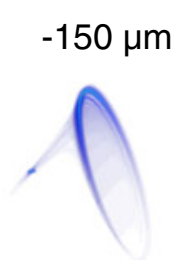

$-100 \mu \mathrm{m}$
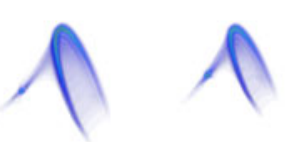

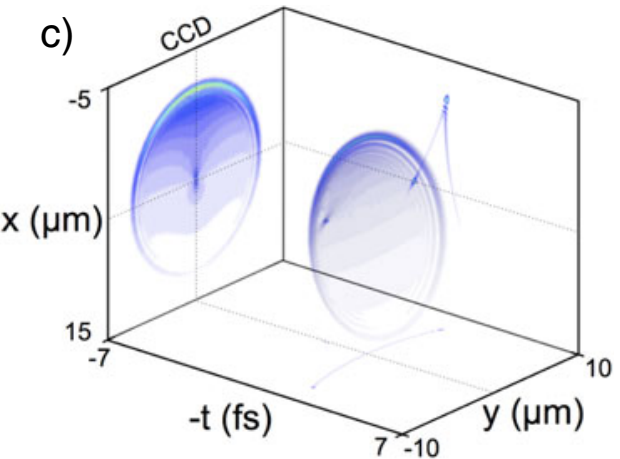

Foyer paraxial
$50 \mu \mathrm{m}$

Figure 3. a) Schéma de la conjugaison optique simulée : un miroir sphérique de focale $100 \mathrm{~mm}$ utilisé avec un angle de champ de $1^{\circ}$ sert à refocaliser des impulsions attosecondes. b) Evolution de l'intensité de l'impulsion attoseconde le long de l'axe de propagation. c) Vue détaillée de l'impulsion $150 \mu \mathrm{m}$ avant le foyer paraxial. Les projections dans les plans $(\mathrm{x}, \mathrm{t})$ et $(\mathrm{y}, \mathrm{t})$ représentent des coupes de l'impulsion au niveau des traits pointillés. La projection dans le plan $(\mathrm{x}, \mathrm{y})$ correspond à l'image de l'impulsion que l'on obtiendrait sur une caméra CCD.

ces impulsions attosecondes, voire pour les recompresser. On peut dès lors imaginer optimiser des miroirs permettant de donner aux impulsions des formes temporelles souhaitées en fonction des applications.

Nous avons également vu que les aberrations pouvaient déformer et étirer les impulsions, ce qui a été confirmé par les simulations numériques. Cependant, l'existence de tels effets pose beaucoup de questions, notamment sur la sensibilité des méthodes actuelles de caractérisations d'impulsions attosecondes, telles que la méthode RABITT. En effet, ces méthodes recontruisent un signal temporel alors qu'une impulsion réelle est toujours spatiotemporelle. Mais sachant que du fait des aberrations, l'impulsion à caractériser n'est pas la même en chaque point, on peut légitimement se demander à quoi correspond l'impulsion temporelle reconstruite. Nous travaillons actuellement sur la question. Enfin, seule une caractérisation spatiotemporelle complète des impulsions attosecondes permettrait de connaître l'influence réelle des aberrations. De telles méthodes de caractérisation existent déjà pour les impulsions infrarouges femtosecondes [18], et leur transposition dans le domaine attoseconde serait un beau défi.

\section{Remerciements}

Nous remerçions S. Nannarone, N. Mahne et A. Giglia pour leur assistance sur le synchrotron ELETTRA, T. Auguste et P. Breger pour leur aide lors des expériences menées au CEA. Nous remercions également le support financier apportés par EU- FP7-ATTOFEL, ANR-09-BLAN-0031-01 and RTRA- Triangle-2008-045T. 


\section{Références}

[1] P. M. Paul, E. S. Toma, P. Breger, G. Mullot, F. Augé, Ph. Balcou, H. G. Muller, and P. Agostini, "Observation of a train of attosecond pulses from high harmonic generation" Science 292, (2001) 1689-1692.

[2] M. Hentschel, R. Kienberger, C. Spielmann, G. A. Reider, N. Milosevic, T. Brabec, P. Corkum, U. Heinzmann, M. Drescher, and F. Krausz, "Attosecond metrology", Nature 414, (2001) 509-513.

[3] D. H. Ko, K. T. Kim, J. Park, J. Lee and C. H. Nam, "Attosecond chirp compensation over broadband high-order harmonics to generate near transform-limited 63 as pulses", New J. Phys. 12, (2010) 063008.

[4] E. Goulielmakis, M. Schultze, M. Hofstetter, V. S. Yakovlev, J. Gagnon, M. Uiberacker, A. L. Aquila, E. M. Gullikson, D. T. Attwood, R. Kienberger, F. Krausz and U. Kleineberg, "SingleCycle Nonlinear Optics", Science 320, (2008) 1614-1617

[5] A. McPherson, G. Gibson, H. Jara, U. Johann, T. S. Luk, I. A. McIntyre, K. Boyer, and C. K. Rhodes, "Studies of multiphoton production of vacuum-ultraviolet radiation in the rare gases", J. Opt. Soc. Am. B 4, (1987) 595-601.

[6] M. Ferray, A. L’Huillier, X. F. Li, L. A. Lompre, G. Mainfray, and C. Manus, "Multiple-harmonic conversion of $1064 \mathrm{~nm}$ radiation in rare gases", J. Phys. B 21, (1988) L31-L35.

[7] Y. Mairesse, A. de Bohan, L. J. Frasinski, H. Merdji, L. C. Dinu, P. Monchicourt, P. Breger, M. Kovacev, R. Taïeb, B. Carré, H. G. Muller, P. Agostini, and P. Salières, "Attosecond synchronization of high-harmonic soft x-rays" Science 302, (2003) 1540-1543.

[8] H. G. Muller, "Reconstruction of attosecond harmonic beating by interference of two-photon transitions", Applied Physics B 74, (2002) S17-S21.

[9] Y. Ménesguen, S. de Rossi, E. Meltchakov, and F. Delmotte, "Aperiodic multilayer mirrors for efficient broadband reflection in the extreme ultraviolet" Appl. Phys. A 98, (2010) 305-309.

[10] A.-S. Morlens, R. López-Martens, O. Boyko, P. Zeitoun, P. Balcou, K. Varjú, E. Gustafsson, T. Remetter, A. L'Huillier, S. Kazamias, J. Gautier, F. Delmotte and M.-F. Ravet, "Design and characterization of extreme-ultraviolet broadband mirrors for attosecond science", Opt. Lett. 31, (2006) 1558-1560.

[11] A. Wonisch, Th. Westerwalbesloh, W. Hachmann, N. Kabachnik, U. Kleineberg and U. Heinzmann, "Aperiodic nanometer multilayer systems as optical key components for attosecond electron spectroscopy", Thin Solid Films 473, (2004) 464-465.

[12] A.-S. Morlens, P. Balcou, P. Zeitoun, C. Valentin, V. Laude and S. Kazamias, "Compression of attosecond harmonic pulses by extreme-ultraviolet chirped mirrors", Opt. Lett. 30, (2005) 1554-1556.

[13] C. Bourassin-Bouchet, Z. Diveki, S. de Rossi, E. English, E. Meltchakov, O. Gobert, D. Guénot, B. Carré, F. Delmotte, P. Salières and T. Ruchon, "Controlling sub-100 attosecond beating with phase-optimized mirrors" (soumis).

[14] Z. Bor, "Distortion of femtosecond laser pulses in lenses", Opt. Lett. 14, (1989) 119-121.

[15] M. Kempe and W. Rudolph, "Femtosecond pulses in the focal region of lenses", Phys. Rev. A 48, (1993) 4721-4729.

[16] U. Fuchs, U. Zeitner, and A. Tünnermann, "Ultra-short pulse propagation in complex optical systems", Opt. Express 13, (2005) 3852-3861.

[17] C. Bourassin-Bouchet, S. de Rossi, F. Delmotte and P. Chavel, "Spatiotemporal distortions of attosecond pulses", J. Opt. Soc. Am. A 27, (2010) 1395-1403.

[18] P. Bowlan, U. Fuchs, R. Trebino and U. D. Zeitner, "Measuring the spatiotemporal electric field of tightly focused ultrashort pulses with sub-micron spatial resolution”, Opt. Express 16, (2008) $13663-13675$. 